\title{
The Chinese Vacuum Society
}

\author{
Jianguo HOU \\ University of Science and Technology of China, 96 Jinzhai Road, Hefei, Anhui 230026, P. R. China
}

\begin{abstract}
Overview
The Chinese Vacuum Society (CVS) is a non-profit organization comprising scientists, engineers and technicians from academia and industry who are involved in vacuum science and technology in China. Founded in 1979, the society now has more than 5000 individual members and 250 corporate members in a dozen local vacuum societies. The CVS is the only representative from China in the International Union for Vacuum Science, Techniques and Applications (IUVSTA). Since joining the IUVSTA in 1983, the CVS has played an active role in various union activities that have helped to foster internationalization and to develop links with other national vacuum societies including the Vacuum Society of Japan. This will culminate in the CVS hosting the 18th International Vacuum Conference (IVC-18) in Beijing in 2010.
\end{abstract}

\section{What is the Chinese Vacuum Society's role within the scientific community?}

For the past 30 years the society has played a committed and active role in promoting the development of vacuum science and technology. It serves as an important platform for knowledge exchange, science popularization and technical consulting through the coordination of academic meetings, workshops and technical training courses, and through the publication of associated material in the society's magazine and on its website. The CVS also acts as a bridge between research institutions and vacuum companies, promoting the technical transfer of scientific information and thus ultimately contributing to China's economic development.

What are the main challenges facing the members of the CVS?

For those members working in academia, the main focus is to continue carrying out innovative vacuum-related research (including the development of instrumentation) and to publish high-quality papers that are recognized by the rest of the world. It is also important to be aware of the application perspective of this research in order to promote knowledge transfer of scientific achievements for technological industrialization. The latter is particularly relevant for those members working in industrial R\&D. The main challenge for them is to improve the quality of vacuum products so that these meet international standards, for example in product reliability. It is also important that CVS members in both academia and industry design and manufacture high-end vacuum systems based on their own intellectual property.

\section{How does the Chinese Vacuum Society plan to develop?}

The CVS is a vibrant and energetic organization. For its continued success I would like to see it focus on five key areas.
- Improving member services such as annual meetings, the society magazine and website, technique consultancy and vacuum exhibitions that offer potential business opportunities.

- Developing vacuum science and technology education initiatives like workshops and technical training courses.

- Promoting internationalization and regional co-operation by hosting international conferences such as IVC-18.

- Establishing vacuum-related engineering standards and qualification schemes for those working in the vacuum industry.

- Encouraging co-operation between vacuum companies to promote technology transfer and the industrialization of vacuum technologies.

\section{What is the current state of vacuum science and technology in} China?

Though developing rapidly, a technology gap still remains between China and Japan as well as the rest of advanced countries. In recent years, however, China has made great progress in vacuum science and technology, both in fundamental research and applied technology. In academia, high-quality and high-impact papers relating to surface science and nanoscience are beginning to appear with more regularity in prestigious international journals such as Nature and Science, with work in fields such as molecular self-assembly and scanning tunneling microscopy partly because nanotechnology is attracting the attention of the international community. In industry, with an ever-increasing number of Chinese vacuum companies, output and production values continue to grow rapidly, particularly for low- and medium-end vacuum products. Ever-improving technical expertise means that the specification and quality of vacuum-pump products are now becoming comparable to those from abroad. Nevertheless, there still exists room for improvement in the quality of vacuum products built in China, particularly those for use in applications requiring reliability and high performance.

\footnotetext{
What are the biggest growth areas in China for commercial vacuum organizations?

In recent years the vacuum industry in China, along with the nation's economy, has grown rapidly. Currently, the biggest growth areas for commercial organizations are in the fields of vacuum-generating equipment and supplementary systems such as those for leak detection and coating technologies. The main application areas for these products being aviation, astronautics, and the chemical and mechanical industries. The CVS, together with the Chinese Vacuum Equipment Association, has worked hard to support these endeavours, for exam-
} 
ple by organizing various vacuum-related symposiums and workshops to serve as a platform for academic exchange, and by offering technical training for universities, research institutes and commercial organizations. It has also helped to establish joint R\&D programmes between universities and companies. All of these efforts help to promote the development of vacuum technology in China, to speed up the development cycle of vacuum products, and ultimately boost the volume of sales of such products.

What is the future of vacuum science and technology in China?

As the Chinese economy continues to grow, one expects similar growth in vacuum science and technology. To achieve sustainable economic development imposes a growing demand for advanced technology and high-performance products. There is a great opportunity for China to play a vital role in hitech areas such as the electronics industry, for example in the development of optoelectronic devices. With regard to the manufacture of vacuum products, the gap between the rest of world and China is shrinking. China will soon become a major player in these fields and vacuum companies will be more involved in international competition. With such globalization, it will become increasingly important to make sure that intellectual property rights are respected and protected. The coalition of Chinese vacuum companies will be an inevitable trend, to allow firms to compete with overseas companies both abroad and in the home market. The Chinese Vacuum Society, as a tie of friendship and a bridge of trade, will do its best to harmonize such competition and thus help promote the worldwide development of vacuum science and technology.

Biography: Jianguo Hou has been the president of the CVS since 2004. He is professor of physics and chemistry at the University of Science and Technology of China, where he is President. His current research interests include nanomaterials, nanodevices and single-molecule science. 